1994 EQEC/CLEO-Europe

\section{First Event a Landmark Success}

With over 2200 participants, the first joint European Quantum Electronics Conference/ Conference on Lasers and Electro-Optics (EQEC/CLEO-Europe) held at the RAI Conference Centre, Amsterdam (28 August - 2 September 1994) proved to be a major success.

EQEC/CLEO-Europe is a new initiative aimed at strengthening the interplay between public-sector R\&D and industrial development in one of the fastest growth areas of modern technology. In the US, CLEO organized in conjunction with the Quantum Electronics and Laser Science conference has successfully bridged the traditional gap between private and public R\&D, notably by featuring a large technical exhibit in addition to the technical conference itself.

The link between academia and industry has historically been weaker in Europe than in the US. This was the main reason the Quantum Electronics and Optics Division (QEOD) of EPS decided to enter into a collaboration with sister organizations in the US to strengthen the impact of the Divisional EQEC event (organized biannually in Europe since 1986). The success of the Amsterdam meeting shows that this motivation was fully justified.

\section{Integrating Research and Technology}

EQEC and CLEO-Europe each have their distinct character, with EQEC covering the fundamental aspects of quantum electronics and CLEO-Europe primarily addressing applied area of lasers and electro-optics. The two distinguished European scientists who spoke during the single EQEC/CLEO-Europe plenary session of the joint conference nicely illustrated the linking of basic research and advanced technology. Both reviewed major recent developments, with $\mathrm{H}$. Walther (MaxPlanck Institute, Garching) discussing micromasers and associated studies of phenomena in quantum optics while D. Payne (Southampton) spoke on the revolution in telecommunications catalyzed by the adven of high-quality optical amplifiers. These are seemingly well-separated topics. However, over the next decade they will merge as quantum optical effects are predicted to enter the field of optical communication owing to stringent requirements on protocols for secure transmission.

The interplay between fundamental research and state-of-the-art applications was further emphasized in two joint symposia. The one on Quantum Engineering of Photonic Materials featured Y. Yamamoto (Stanford and Tokyo), who spoke on microcavity lasers, and C. Weisbuch (Ecole Polytechnique, Paris) who discussed cavity quantum electrodynamics in multiple quantum-well structures. Both gave clear evidence that optical microcavities represent a fast maturing field (see page 205) with many potential applications, including noise control in optical communications systems. E. Yablonovitch (Los Angeles) reviewed photonic bandgaps and semiconductor electronics. Among several fascinat-

Ove Poulsen is the Director of the Microelectronic Centre, TU Lyngby, Denmark. He co-chaired the EQEC '94 Programme Committee with Elizabeth Giacobino from Paris ing topics, he described an intelligent programmable radar reflector that resembled a fence made of chicken wire.

The second joint symposium dealt with the Production and Application of High Brightness Ultrafast Pulses. This field has wit- nessed a very strong development of both fundamental and applied natures, catalyzed by the advent of new, powerful, short-pulse lasers. S. Szatmari (Göttingen) discussed table-top, high-power femtosecond excimer lasers, while three speakers covered highbrightness X-ray sources, among them $E$. Turcu (Rutherford Appleton Laboratory) and C. Schank (Berkeley) who reviewed applications of laser-generated X-rays for lithography and microscopy. This application is potentially very important because it is capable of introducing table-top X-ray sources into the electronics industry. The symposium also dealt with more fundamental issues, notably high harmonic generation in various media. Impressive progress was reported on the generation of tuneable XUV radiation featuring parametric mixing. The broad interest in high power and short-pulse lasers was accentuated by two well-attended tutorials given by $B$. Krupke (Lawrence Livermore National Laboratory) and F. Krausz (Vienna) that set the stage for the symposia presentations which followed.

Aside from high power and short pulse lasers, CLEO-Europe included highlights in the fields of waveguide- (fibre-) based lasers, with A.C. Tropper (Southampton) and R. Wyatt (British Telecom) giving invited talks on the rapid developments, including up- and down-conversion. On the EQEC side, the interesting properties embedded in the transverse degrees of freedom of laser fields (i.e., transverse optical pattern formation) was discussed by several speakers, among them G.-L. Oppo (Strathclyde) who showed video recordings of calculated spontaneously generated patterns.

\section{EQEC Symposia Attracted Many}

Two EQEC symposia, Cold Atoms and Atom Optics and Interferometry, attracted large crowds on the last day of the joint conference. The leading scientists in these extremely active research areas gave a series of talks of a very high quality. The field of cold atoms is developing rapidly with the custom design of optical lattices for cold atoms. G. Grynberg (Paris) and T.W. Hänsch

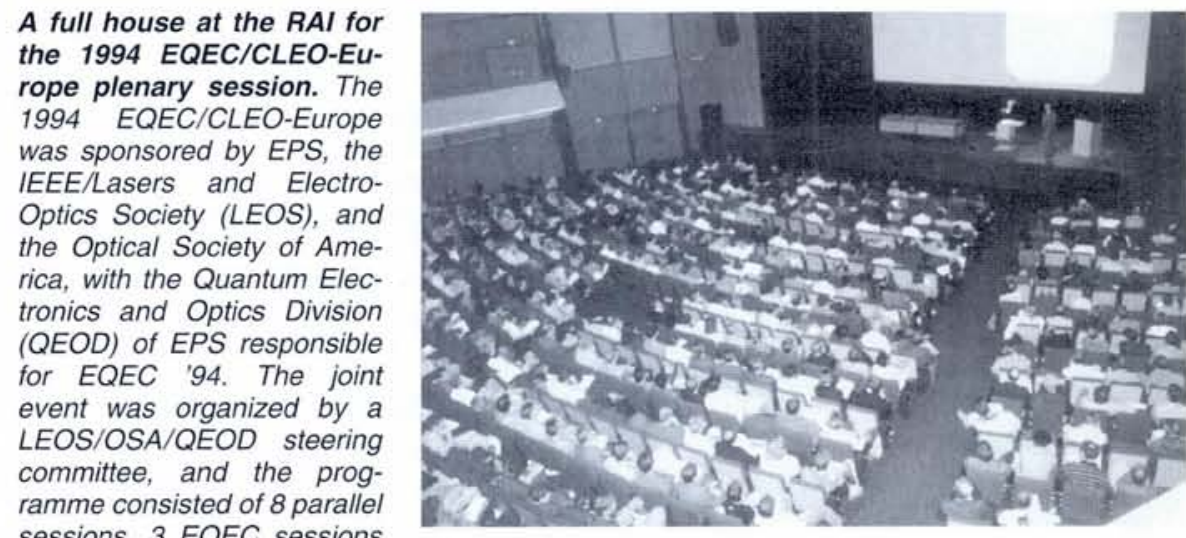
sessions, 3 EQEC sessions

and 5 CLEO-Europe sessions. There were 2200 participants, including some 1500 for the conferences themselves and 300 who visited the industrial exhibition. Conference participants could register either for EQEC (40\% chose this) or for CLEO-Europe, but both groups received the technical digests for the two events. A total of 1500 papers (600 for EQEC) were submitted from nearly 50 different countries: some $40 \%$ were selected for oral presentation, $30 \%$ were given as posters and $30 \%$ were rejected. In addition, nearly 60 invited papers in the EQEC part and 90 invited papers in CLEO-Europe ensured a high scientific quality of this first major European event in quantum electronics and lasers. The success of the meeting was greatly enhanced by the early acceptance of many of the invited speakers who thus lent their prestige. 


\section{QEOD Board Elects New Chair}

M. Ducloy (Paris) and O. Poulsen (Lyngby), on the right. Professor Mlynek reported during the General Meeting of the Division held at the end of the EQEC/CLEOEurope joint plenary session that Ducloy would be taking over from him next April. He also summarised the Division's wide-ranging activities including: plans for a "who's who" (the last was published in 1984); the Division newsletter in Optics Communications; a possible EPS prize in quantum electronics;

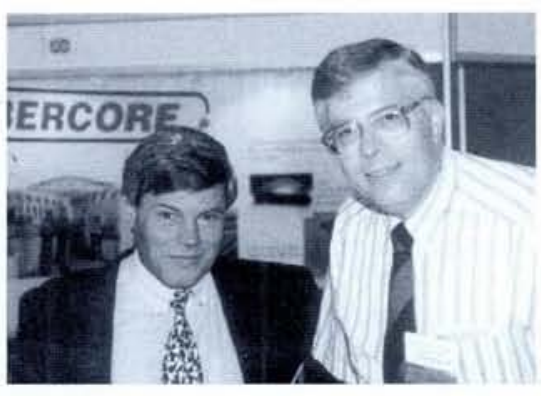
conferences, notably the very successful European Research Conference in quantum optics, and of course EQEC in its new format; and finally, efforts to enhance relations with industry, the European Community and with the European Optical Society. Many scientists and engineers working in optics attended the 1994 EQEC/CLEO-Europe, thereby illustrating the optical community's high level of interest.

(Garching) discussed in detailed design criteria for the optical potentials in both two- and three-dimensions, and gave examples of unexpected cooperative effects resembling phenomena found in the solid state. The basic physical phenomena behind laser cooling of

free and lightly bound atoms were reviewed by M.J. Phillips (National Institute of Science and Technology, Washington), a well-known pioneer of laser cooling, in an exceptionally clear presentation.

The EQEC symposium on Atom Optics

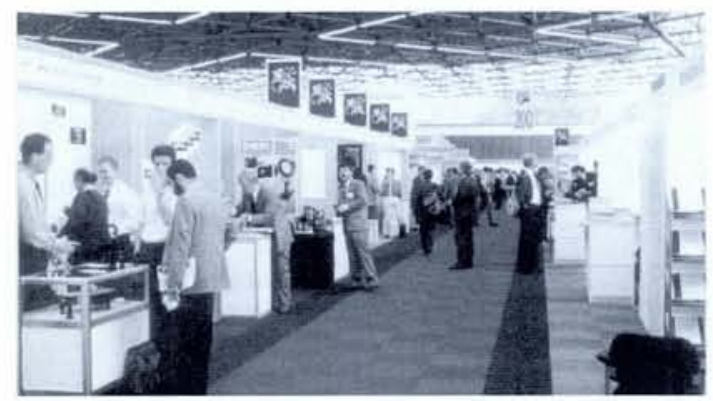

Over 200 companies represented. Aside from the conferences themselves, the 1994 EQEC/ CLEO-Europe featured a large, three-day commercial exhibit where more than 200 companies displayed their latest innovations at 122 stands. The inclusion of a strong technical exhibit was an important part of the first EQEC/ CLEO-Europe. It served as a meeting place not only for science and technology, but also for industrial participants and the large number (over 400) of students who could be inspired by new products and establish new contacts aimed at an eventual career in industry.

\section{EQEC/CLEO-Europe HIGHLIGHTS}

\section{MONOLITHIC MINIATURE RESONATORS}

\section{Manipulating the Electromagnetic Field}

A major success of quantum optics in the last decade has been the experimental demonstration that the quantum fluctuations of light can be modified: the fluctuations in one quadrature (the amplitude of the cos[qt] or $\sin [q t]$ component of the electric field) can be decreased ("squeezed") below the vacuum level, while those in the orthogonal increase correspondingly in order to satisfy the Heisenberg uncertainty relation. Squeezed light can be produced by subjecting a light mode to a nonlinear interaction of the type that gives rise to second-harmonic and subharmonic generation (so-called optical parametric oscillation).

Unfortunately, squeezed light is fragile: its nemeses are the ubiquitous propagation losses. To generate strongly squeezed light one must provide an environment in which the nonlinear interaction can act while the losses play a secondary role.

At present, the experimental emphasis relies on developing reliable and efficient sources of strongly squeezed radiation for a number of applications. An "all solid-state" approach has been adopted at the University of Constance by combining diode laser-pumped solid-state lasers and monolithic nonlinear resonators (see photograph). Roundtrip losses as low as $0.2 \%$ have been routinely achieved.

In a first experiment, a continuous-wave, single-mode wave from a frequency-tuneable Nd:YAG laser was resonantly injected into a

\section{EQEC/CLEO-Europe}

The 1996 joint European Quantum Electronics Conference/Conference on Lasers and Electro-Optics - Europe will be held in France or Germany in September 1996. In view of rapid developments, there will be two new EQEC session (on atom optics and on cold atoms). The CLEO-Europe general chairs are D.C. Hanna and J.-P. Huignard; the EQEC general chairs are $\mathrm{O}$. Poulsen and E. Giacobino.

For information, contact: $\mathrm{O}$. Poulsen, $\mathrm{Mi}-$ croelectronic Centre, TU Lyngby, DK-2800 Lyngby, Tel./fax: +45 9346 10/45 887762 ; op @ mic.dtu.dk. and Interferometry had the same rare qualities as the symposium on cold atoms by offering a well-balanced series of talks by active researchers representing state-of-theart activities in a rapidly growing field. The world of Newtonian physics was successfully merged with quantum physics by J. Dalibard (Paris), who described the motion of lasercooled caesium atoms in a gravitational cavity (i.e., a cavity that apparently has no magnetic field present). The extreme precision inherent in using laser-cooled free atoms in combination with atom interferometry was convincingly discussed by $\mathrm{J}$. Helmcke (Physikalisch-Technische Bundesanstalt), and the fundamental measurement limits were reviewed by T. Pfau (Constance). A highlight of the symposium was an introduction to the (new) topic of non-linear atom optics by $P$. Meystre (Tuscon). The main problem in defining non-linear effects lies in the intricate interaction between atoms in optical fields.

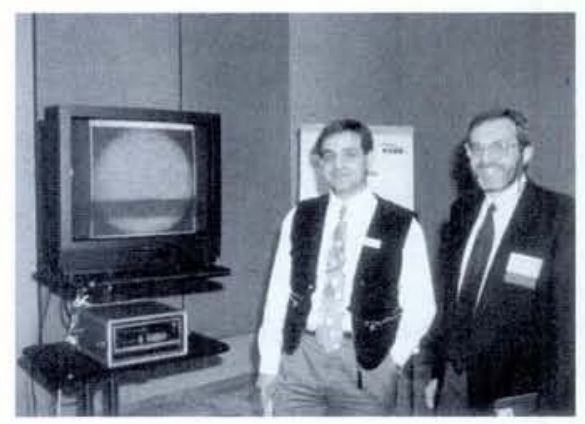

G.-L. Oppo (Strathclyde), on the left, demonstrating his video of transverse optical patterns in laser fields, with L.A. Lugiatio (Milan) who takes over from E. Giacobino (Paris) as the QEOD Secretary next April.

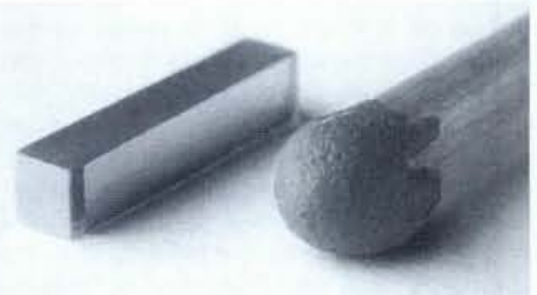

Monolithic nonlinear optical resonator. Shown is a resonator fabricated out of a lithium-niobate single crystal whose ends are accurately polished into a spherical shape. Multilayer dielectric coatings are sputtered directly onto the end faces of the crystal.

monolithic resonator. The high circulating power that builds up within the cavity is converted to the harmonic wave (532 $\mathrm{nm}$ wavelength) which immediately escapes from the resonator. When the device is operated in an input-power regime such that the external power conversion efficiency is high, the harmonic wave exhibits intensity noise below the vacuum level. A reduction of $30 \%$ has been measured, with more than $50 \mathrm{~mW}$ of optical power [Paschotta et al., Phys. Rev. Lett. 73 (1994) 3807]. This source is remarkably stable over many hours, and represents a very simple and reliable way of producing squeezed light.

Besides bright squeezed light, the generation of squeezed vacuum is also of great 


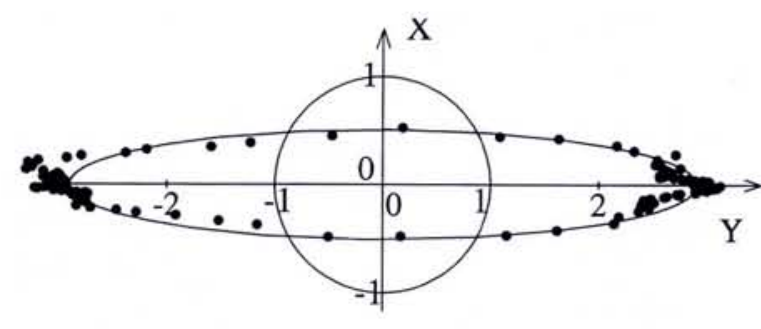

"Squeezing ellipse", showing the measured reduced and excess quantum noise in the electric field quadratures of a squeezed vacuum. The circle indicates the usual zero-point vacuum fluctuations. The ellipse area exceeds the circle area owing to the effect of the small, but finite, system losses.

interest for a number of applications. Squeezed vacuum is characterized by a vanishing expectation value of the electric field, but reduced fluctuations in one quadrature. A parametric amplifier is used to generate light of this type. The monolithic resonator employed in the "squeezing" experiment was operated "in reverse", namely pumped by a $532 \mathrm{~nm}$ wave. When the pump power was below the threshold of $28 \mathrm{~mW}$, squeezed vacuum was generated, with a measured $55 \%$ reduction in quantum noise power in one quadrature (see figure).

While squeezed vacuum of this sort is obtained when "normal" vacuum at $1064 \mathrm{~nm}$ enters the cavity, it is also possible to inject a weak laser beam, giving rise to low-power squeezed light. In this arrangement, a $65 \%$ quantum noise reduction was observed.

The systems described are compact, efficient, and highly reliable. The all-solid-state, monolithic, approach is therefore well suited for applications of squeezed light in complex experiments, such as gravitational wave detection. The development of low-loss nonlinear resonators is also important for other quantum-optical experiments. Moreover, the requirements that benefit strong squeezing also lead to highly efficient frequency conversion. More than $80 \%$ power conversion was obtained with the monolithic resonator in harmonic generation as well as in subharmonic generation with a pump power four times above oscillation threshold.

S. Schiller, J. Mlynek University of Constance

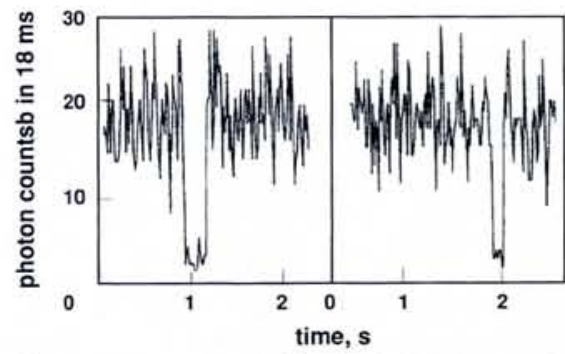

Fluorescence signal of a single $\mathrm{In}^{+}$ion on the ${ }^{1} S_{0} \rightarrow 3 P_{1}$ line, showing dark periods whenever the metastable ${ }^{3} P_{0}$ level is excited.

used to probe the excitation spectrum of the ion.

[1] Hollemann G., Peik E. \& Walther H., Phys. Rev. A 49 (1994) 402.

E. Piek, G. Hollerman, H. Walther MPI for Quantum Optics, Garching University of Munich fourth harmonic of a Nd:YAG laser line. Thus an ultrastable frequency quadrupled diodepumped Nd:YAG laser operating at $946 \mathrm{~nm}$ is
The prospective clock transition is the ${ }^{1} \mathrm{~S}_{0}$ $\rightarrow{ }^{3} \mathrm{P}_{0}$ line at $236.5 \mathrm{~nm}$, a very weak electromixing of ${ }^{3} \mathrm{P}_{0}$ with ${ }^{3} \mathrm{P}_{1}$ and ${ }^{1} \mathrm{P}_{1}$. We recent to be only $1.1 \mathrm{~Hz}$ [1], corresponding to a dark period in the single-ion fluorescence signal on the cooling transition. Samples of fluorescence signal data exhibiting quantum jumps are shown in the figure. The frequency candidate for these applications. We cool it by exciting the intercombination line $5 s^{2}{ }^{1} s_{0} \rightarrow 5 s 5 p^{3} P_{1}$ at a wavelength of 230.6 be possible using sideband laser cooling.

\section{PHOTON CONTROLLED STRUCTURES Breakthroughs at Hand}

The special 1994 EQEC/CLEO-Europe joint session Quantum Engineering of Photonic Materials as well as several presentations indicated that photonmode control in optical microcavities and bandgap materials has made major advances recently. Claude Weisbuch (Ecole Polytechnique, Paris) reports.

During the 1994 EQEC/CLEO-Europe joint symposium, E. Yablonovitch (Los Angeles) insisted on the huge device applications which can be forecast for the emerging field of photon-mode control in semiconductor microcavities or photonic bandgap (PBG) materials [1]. Moreover, recent implementations which rely on "photonic atoms" have turned out to be easier to fabricate than those originally proposed. It is therefore not surprising that major advances [2] were reported at the 1994 EQEC/CLEO-Europe.

\section{Couple-Mode Luminescence}

The driving force in the the field of photonmode control is the ability to manipulate spontaneous light emission, photon-matter interaction, beam performance, etc. via the selective enhancement or suppression of photon modes in wavelength-sized, post-

Fig. 1 - Weak light-matter coupling. Schematic illustrations (a, left) of optical microcavities with various dimensionalities of the photon modes established using localized (e.g., metallic) mirrors. Photonmode densities ( $b$, right) as a func-
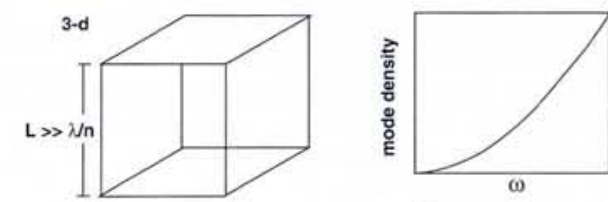

$2-d$
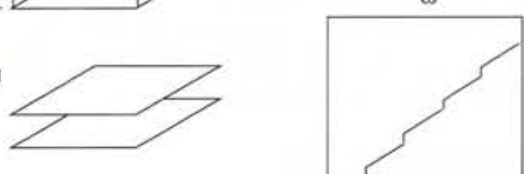

$1-\mathrm{d}$

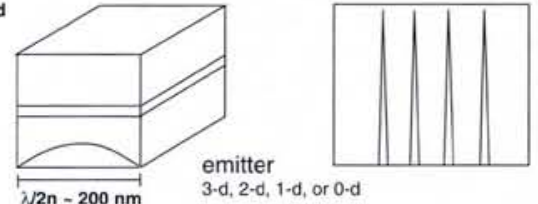

tion of the optical frequency for each of the three types of microcavities. In the 2-d (planar) or 3-d (box-like) cavities, one or three dimensions of the cavity are of the order of the optical wavelength $\lambda$ divided by the effective index of refraction $n$. Emission spectra correspond directly to the mode densities with, for example, sharp emission lines for 0-d cavities.

shaped structures (microcavities) or regular structures (PBG materials). The regimes of weak and strong light-matter coupling are being investigated. In the former, the modification of light emission that can be achieved by controlling the photon modes is fully described by adjusting the photonic densityof-states (Fig. 1) and, in the case of sponta- neous emission, by the resulting modification of Fermi's golden rule. In the strong-coupling regime, the interaction of a selected and enhanced photon mode with the material's excitations is so strong that the perturbation approach breaks down: the materials system and the photon-mode system interact coherently with one another, giving rise to a Rabi 
oscillation of the coupled photon-matter system between its two states, where one of the two systems, material or photon, is in the excited state while the other is in the ground state, and vice-versa.

C. Weisbuch (Ecole Polytechnique, Paris) described the strongly-coupled system realized by inserting semiconductor quantum wells in a planar Fabry-Perot microcavity consisting of monolithically-integrated, directly-grown, quarter-wave layer stacks of mirrors (a so-called distributed-Bragg reflector or DBR) sandwiching a spacer layer (Fig. 2a). The most recent work [3] deals with the observation and understanding of coupledmode luminescence. The in-plane momentum dispersion curves of cavity polaritons (the coupled-mode dispersion) has been determined from a detailed analysis of the angular-resolved emission and is found to agree with the predicted curves (Fig. 2b). One therefore now understands the basic aspects of cavity-polariton emission.

There remain, however, some aspects that need further work. In particular Y. Yamamoto (Stanford and Tokyo) discussed the effects observed at high intensity with stronglycoupled semiconductor quantum wells in microcavities. He modified the usual lightmatter Hamiltonian describing the photon field, the material system, and their interaction by inserting boson operators. The solution led to sidebands instead of the usual Rabi doublet, However, this attributing of luminescence sidebands to "Rabi sidebands" originating in the boson nature of the excitons is at variance with the normal fermionic behaviour of electrons in atoms.

Besides the special session, several presentations also dealt with photon-controlled structures. F. Yang et al. (Cardiff) reported a $25 \%$ improvement of the threshold of horizontal Fabry-Perot lasers using a planar microcavity to suppress unwanted spontaneous emission into vertical modes. Stanley et al. (EPF-Lausanne) demonstrated stronglycoupled multiple microcavities, whereby one couples a material system (quantum wells) with several photon-coupled microcavities. Being able to independently specify the coupling strength between the various oscillators gives a welcome handle on the concept of variable or heterogeneous coupling (the cavities, for instance, could associate different materials and functionalities)

\section{High Efficiency LEDs}

But the major issue in the field is to improve light-emitting devices (LEDs). Research is active world-wide and could soon provide a major breakthrough in the high-efficiency generation of light. One of the most desirable devices is the "zero-threshold" laser, where suppression of spontaneous emission in all photon modes but one, i.e. the laser mode itself, allows continuous switching from spontaneous to stimulated emission (photon number in the remaining mode $>1$ ), with a constant quantum efficiency near unity. Additionally, the emission process would become fully deterministic, which could lead to photon emission that is limited by Johnson noise instead of the much stronger shot noise arising from the coexistence of random spontaneous emission with laser emission. De Neve et al. (IMEC, Gent) reported an important step towards the realization of high-efficiency LEDs, possibly
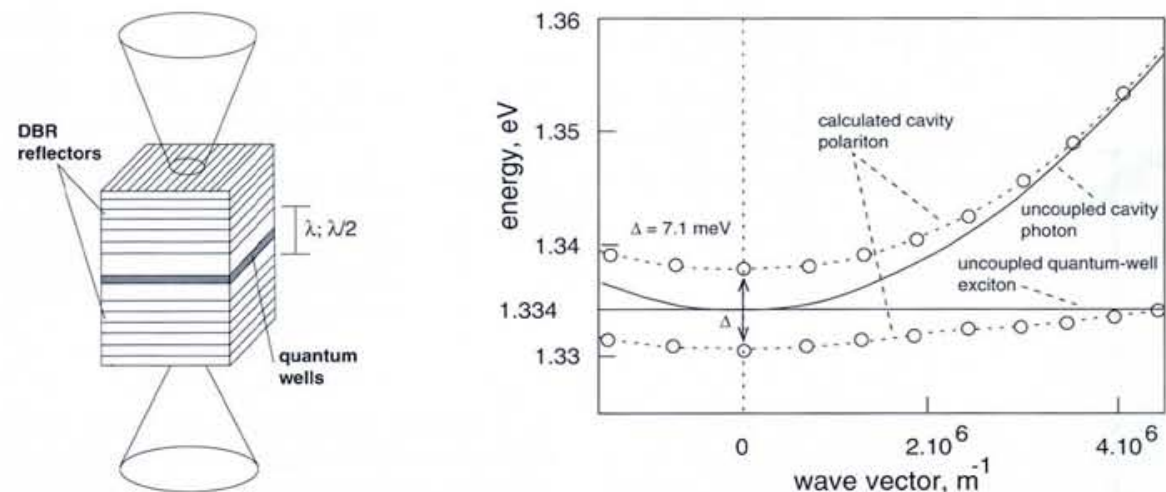

Fig. 2 - Polaritons in a planar semiconductor microcavity. a, left) Semiconductor quantum wells are inserted in a planar Fabry-Perot microcavity consisting of monolithically-integrated, directly-grown, quarter-wave layer stacks of mirrors (a so-called distributed-Bragg reflector or $D B R$ ) sandwiching a spacer layer with a thickness of $\lambda / 2$ or $\lambda$. Strongly coupled behaviou arises when the cavity resonant photon mode parallel to the layers resonates with the quantum-well exciton. $b$, right) In-plane dispersion of the coupled exciton-cavity photon mode as determined from angular-resolved luminescence measurements. The experimental data points are accurately described by the coupled-mode dispersion (lines), the so-called cavity polariton, calculated by assuming that the excitons and the resonant cavity form a simple system of two oscillators coupled through the light-matter interaction.

zero-threshold lasers, by carefully designing a planar microcavity to give an external quantum efficiency of $6.2 \%$ (recently extended to $10.5 \%$ ).

[1] Yablonovitch E., J. Opt. Soc. Amer. B 10

\section{Austrian Textbook Wins Amaldi Prize}

Reconciling our everyday world with the complex logical construction based on empirical principles and mathematics that allows natural phenomena to be modelled is seen by many as the challenge facing secondary school teachers. The winner of the 1993 Amaldi International Prize for a High-School Physics textbook addresses this problem by splitting the physics course into its essential parts (as four, short compact, volumes on basic knowledge) complemented by series of 12 theme-oriented books dealing with applications in daily life. The books also introduce some novel but useful devices, such as logos asking the student to do something (e.g., "think about ..."), ideas for experiments for the at home, and English translations of technical terms.

The winning textbook is called Physikcompact published by Verlag Hölder-PilcherTempsky (Vienna), Austria's largest publisher of maths and physics textbooks. The authors are Albert Jaros, Alfred Nussbaumer, Peter Nussbaumer, Hansjoerg Kunze, Leopold Mathelitsch, Robert Hofstetter, and Hans Haimo Tentchert. R. Hofstetter teaches physics and philosophy at Vienna University and in a gymnasium and L. Mathelitsch is Professor of Theoretical Physics at the Technical University, Graz. The other authors are all teachers in gymnasia, with the two Nussbaumers being twin brothers.

Physik-compact was published to coincide with the introduction of Austria's new physics curriculum in 1989, which the authors were heavily involved in defining. The four basic volumes (Baiswissen) are only sold in Austria, but some 1500 of the theme-oriented books (Themenhefte) have been sold in Germany. The Prize Jury felt the contents were flexible enough to suit curicula in different countries. This may encourage the publication of translations, for which there are no plans at present.
(1993) 283; J. Mod. Optics 41 (1994) 171

[2] See, e.g., Slusher R.E. \& Weisbuch C. Solid State Commun. 92 (1994) 149.

[3] Houdre R. et al., Phys. Rev. Lett. 73 (1994) 2043

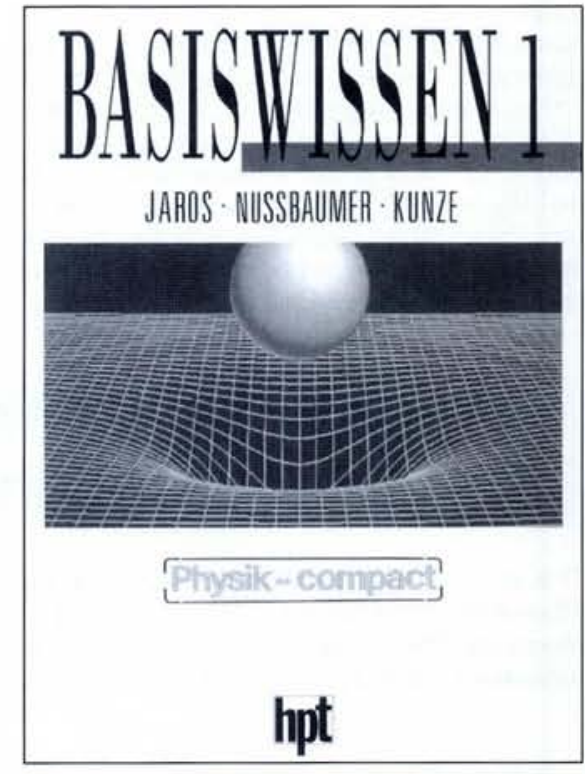

Physik-compact

4 main books ( 452 p.): AS515.-

12 theme-oriented books (464p.): AS 597.Publisher: Verlag Hölder-Pichler-Tempsky Jochen-Rindt-Strasse 11, A-1232 Vienna Tel.: +43-1-4389930

The 1993 Amaldi Prize, the first ever, was awarded by the Edoardo Amaldi Foundation, Piacenza, with EPS sponsorship. It comprised 20000 ECU given to the authors and publishers of a high-school physics textbook first printed in Europe between 1 January 1987 and 30 September 1993. A total of 47 books were submitted out of an estimated $60-70$ potential candidates, there being none in French and relatively few in German. The next prize will be awarded in a few years time. 